\title{
A study to compare the conjunctival flora of non-diabetic individuals with that of diabetic patients
}

\author{
Rajeshkannan R. ${ }^{1}$, Venkatesan M.J. ${ }^{2}$, Ezhilvendhan K. ${ }^{3}$, Rao A.V.R. ${ }^{4}$ \\ ${ }^{1}$ Dr. R. Rajeshkannan, Assistant Professor, Department of Ophthalmology, ${ }^{2}$ Dr. M.J. Venkatesan, Associate Professor, \\ Department of Ophthalmology, Melmaruvatthur Adiparasakthi Institute of Medical Sciences, Melmaruvathur, \\ Kanchipuram District, Tamil Nadu, ${ }^{3}$ Dr. K. Ezhilvendhan, Professor \& HOD, Dept. of Ophthalmology, ${ }^{4}$ Dr. A.V. \\ Raghavendar Rao, Assistant Professor, Department of Microbiology; 1,3,4authors are affiliated with Vinayaka Mission's \\ Kirupananda Variyar Medical College \& Hospital, Vinayaka Mission's Research Foundation, Deemed to be University, \\ Salem, Tamil Nadu, India.
}

Corresponding Author: Dr. M.J. Venkatesan, Associate Professor, Department of Ophthalmology, Melmaruvatthur Adiparasakthi Institute of Medical Sciences, Melmaruvathur, Kanchipuram District, Tamil Nadu, India. E-mail ID: drvenkatesan.mj@gmail.com

\begin{abstract}
Background: The conjunctiva is a thin, transparent and humid membrane which covers the sclera and inner surface of eyelids. The tears film helps to moist the conjunctiva. In a healthy person, surface tissues such as skin and mucous membranes are constantly in contact with environmental organisms and become colonised by various micro-organisms, bacteria and fungi which are referred to as normal flora. Aim: To compare the conjunctival flora of non-diabetic individuals with that of diabetic patients. Methodology: A prospective study for comparison of conjunctival flora of 50 non-diabetic individuals with that of 50 diabetic individual patients without any pre-existing conjunctival pathology attending the Ophthalmology Out Patient Department in Vinayaka Mission's Kirupanada Variyar Medical College and Hospital, Salem, Tamil Nadu, which is 560 bedded hospital having all inpatients and outpatient services. Conjunctival swab was collected from each patient and inoculation of conjunctival swab for microscopy and culture and sensitivity was done. Identification of Bacteria were made using Standard Bacteriological methods. Result: We found a significant difference in bacterial isolation rate between the diabetic and non-diabetic groups. Conclusion: Organisms such as coagulase negative staphylococcus, gram negative bacteria such as E. coli, klebsiella and bacteroids were found to be more common among the diabetic patients than that of non-diabetic patients.
\end{abstract}

Keywords: Conjunctival flora, Standard Bacteriological methods, Coagulase negative staphylococcus, Escherichia coli

\section{Introduction}

The conjunctiva is a thin, transparent, smooth and humid membrane that covers the sclera and inner surface of eyelids [1]. The conjunctiva is kept moist and healthy by tear which contains lysozyme, IgA, IgG, âlysine, lactoferrin, complement and multiple antibacterial enzymes [2]. In a healthy person, surface tissues such as skin and mucous membranes are constantly in contact with environmental organisms and becomes colonized by various micro-organisms which are referred to as normal flora [3]. Bacteria and fungi are considered as normal flora of conjunctiva whereas viruses and parasites are not considered as the members of the normal flora [4].

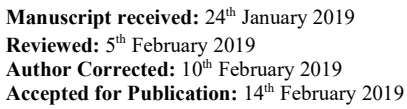

The predominant microorganisms of conjunctiva are Staphylococcus epidermidis (30-80\%), Diphtheroids (5$83 \%$ ), Micrococcus sp. (1-28\%) and Staphylococcus aureus (3-25\%). In addition, Streptococcus pyogenes (0$3 \%$ ), Streptococcus pneumoniae (0-3\%), Streptococcus viridans $(0-1 \%)$, Moraxella catarrhalis (2-5\%), Haemophilus influenzae (0-1\%), Klebsiella sp. (0$0.5 \%)$, Escherichia coli $(0-1 \%)$ and Pseudomonas aeruginosa (0-2\%) are occasionally found [5].

Normal conjunctival flora remains relatively consistent among human populations. However, it may be altered by a variety of factors including age, immunesuppression, ocular inflammation, dry eye, use of contact lens use, antimicrobials, surgery, external exposure, climate and geography. 


\section{Original Research Article}

Some members of the conjunctival flora play a pathogenic role in diabetes mellitus when immune function is compromised, which may lead to serious infection [6].

In diabetes mellitus, some members of the normal conjunctival flora play a pathogenic role when immune function is compromised, which may result in serious infection [7]. It was reported that diabetic patients have higher levels of glucose in their tears than the non- diabetics, which may contribute to the development of ocular infections [8]. Various studies have shown that positive conjunctival flora culture varies from $16.6 \%$ to $65 \%$ [9]. Suto et al. found that Gram-positive cocci formed $67 \%$ of all isolates [10].

The conjunctival flora in diabetic subjects may differ from that in non-diabetic subjects. Therefore, this study was done to analyze the bacterial flora of Type II diabetics in comparison with normal subjects.

\section{Materials and Methodology}

Study Period: January 2016 - June 2017

Source of Data: Patients (Diabetic and Non-Diabetic) attending the Ophthalmology OPD in Vinayaka Mission Medical College and Hospital, Salem between January 2016-June 2017

Sample Size: 100 (50 diabetic and 50 non-diabetic patients)

Study Design: Hospital based, Prospective Study.

\section{Inclusion Criteria}

1. Diabetic and Non-diabetic patients without any pre-existing conjunctival pathology.

\section{Exclusion Criteria}

1. Patients with pre-existing ocular disease including conjunctival pathology

2. Present history of any antibiotic instillations

3. Patients who are wearing contact lens

4. Patients with history of previous invasive ocular surgery

Specimen - wet Brain Heart Infusion (BHI) conjunctival swab from the lower fornix Volume - one swab from each patient

Collection - sterile moist (moistened in BHI broth) cotton wool swab rolled across the lower conjunctival fornix Storage instructions - directly inoculated on the culture media.

Informed verbal consent - obtained from all individuals. A detailed history was taken from the individuals and a thorough ocular surface examination was carried out on the slit lamp to exclude any infections or ocular surface diseases.

Ethical Committee Clearance-This study has been approved by Ethical Committee Board, Vinayaka Mission's Kirupananda Variyar Medical College, Salem

\section{Specimen collection and processing}

1. Inoculation of the conjunctival swab for microscopy was carried out as follows: samples from the conjunctiva were collected by rolling the moistened (BHI broth) swab stick in the lower conjunctival fornix from the medial to the lateral canthus and smearing it directly on the naked glass slide to make a thin film. Naked glass slides were then gram stained and examined under a microscope for micro organisms.

2. Inoculation of the conjunctival swab for culture and sensitivity was carried out as follows: inoculation was done after wetting the swab with BHI broth. The swab was then taken from the lower conjunctival fornix. Inoculation in the Petri dish was done in the following order: chocolate agar, followed by blood agar, followed by Thioglycolate agar.

3. Reading of the Petri dish and the broth was done after 24 and $48 \mathrm{hrs}$ of inoculation after which the dishes were discarded.

4. The questionnaires were completed as per the findings on the Petri dishes and the sensitivity patterns were recorded. Blood Agar (BA) and Chocolate Agar (CA) were used as enriched media.

5. Thioglycolate broth was used as differential medium (for both aerobes and anaerobes). Thio broth was used for anaerobic organisms. BHI and Thio broth were used as enrichment media too. 
6. BA and $\mathrm{CA}$ plates were incubated in a $\mathrm{CO} 2$ jar at $37^{\circ} \mathrm{C}$. $\mathrm{BHI}$ and Thio agar will be incubated at $37^{\circ} \mathrm{C}$. A positive culture was defined as growth on any one of the 4 media used.

7. Colony count was carried out on all positive cultures, classified as light growth (less than 20 colonies ), moderate growth (20 - 100 colonies), and heavy growth ( more than 100 colonies)

8. If no growth was obtained the plates were incubated for another $24 \mathrm{hrs}$. In case of negative cultures, the plates were then discarded. In case of negative growth on solid media, smears were made directly from the broth that showed turbidity (which is an indication of growth). Subcultures were made from both the broths.

9. Identification of the bacteria were made using standard bacteriological methods.

10. All the micro organisms were tested to antibiotics chosen on the basis of the gram stain results. The Kirby Bauer disc diffusion method of sensitivity testing was applied.

Statistical analysis: Age wise distribution of the study population shows that majority of the study subjects in both the diabetes group and the control group were between 50 and 60 years. The mean age of diabetes and control group was 61.1 and 56.8 years respectively. There was no statistical difference in the age group between the two groups. Gender wise distribution of the study subjects shows that the females are more in number in both the groups than the males but the proportion of males and females in both the groups are almost same and there was no statistical significant difference between the two groups.

Table-1: Percentagewise Distribution of the diabetes patients based on their duration of Diabetes

\begin{tabular}{|c|c|c|c|}
\hline Duration of diabetes (in years) & Frequency $(\mathbf{n}=\mathbf{5 0})$ & Percentage & Mean \pm SD \\
\hline $\mathbf{3}-\mathbf{5}$ & 5 & $10 \%$ & \multirow{2}{*}{$8.75 \pm 3.18$} \\
\cline { 1 - 3 } $\mathbf{5}-\mathbf{7}$ & 8 & $16 \%$ & \\
\cline { 1 - 3 } & 14 & $28 \%$ & \\
\hline $\mathbf{9}-\mathbf{1 1}$ & 11 & $22 \%$ & \\
\hline $\mathbf{1 1}-\mathbf{1 3}$ & 12 & $24 \%$ & \\
\hline
\end{tabular}

Table 1 shows the distribution of diabetes patients based on the duration of diabetes. It is seen from the table the mean duration of diabetes among them was 8.75 years, with a mean duration of 3 years and the maximum duration of 12 years.

Table-2: Mean and SD of RBS, FBS and PPBS among the diabetes patients

\begin{tabular}{|c|c|c|}
\hline Blood sugar parameters & Mean $(\mathbf{m g} / \mathbf{d l})$ & SD \\
\hline RBS & 176.3 & 17.14 \\
\hline FBS & 157.2 & 10.11 \\
\hline PPBS & 230.3 & 31.2 \\
\hline RBS among control group & 92.3 & 9.0 \\
\hline
\end{tabular}

Table 2 shows the mean and SD of the various glucose parameters among the study subjects. It is seen from the table the fasting, random and post-prandial blood glucose values among the diabetes patients were $157.2 \mathrm{mg} / \mathrm{dl}, 176.3 \mathrm{mg} / \mathrm{dl}$ and $230.3 \mathrm{mg} / \mathrm{dl}$ respectively. The random blood sugar level among the non-diabetes group was $92.3 \mathrm{mg} / \mathrm{dl}$ (Table No.-2)

Table-3: Microscopy findings of the conjunctiva flora among the study subjects

\begin{tabular}{|c|c|c|c|}
\hline $\begin{array}{c}\text { Microscopy findings of } \\
\text { conjunctiva }\end{array}$ & Diabetes group $(\mathbf{n = 5 0 )}$ & $\begin{array}{c}\text { Control group } \\
(\mathbf{n = 5 0})\end{array}$ & \multirow{2}{*}{ P value } \\
\cline { 1 - 3 } Gram positive cocci & $34(68 \%)$ & $25(50 \%)$ & \multirow{2}{*}{$<.001$} \\
\hline Gram negative bacilli & $10(20 \%)$ & $5(10 \%)$ & \\
\hline No growth & $6(12 \%)$ & $20(40 \%)$ & \\
\hline
\end{tabular}

P value derived by applying chi-square test

Table 3 shows the microscopy of the conjunctiva flora among the study subjects. Among the diabetes group $68 \%$ of the study subjects had shown the presence of gram-positive cocci, whereas among the control group only $50 \%$ had shown the presence of gram-positive cocci and $40 \%$ of them did not show any organism in the microscopy and this difference was found to be statistically significant $(\mathrm{p}<.05)$. 
Table-4: Culture findings of the conjunctiva flora among the study subjects

\begin{tabular}{|c|c|c|c|}
\hline Culture findings of the conjunctiva flora & Diabetes group (n=50) & Control group (n=50) & P value \\
\hline Staphylococcus aureus & $14(28 \%)$ & $15(30 \%)$ & $12(24 \%)$ \\
\hline CONS & $18(36 \%)$ & $1(2 \%)$ & \multirow{2}{*}{$<.001$} \\
\hline E. coli & $4(8 \%)$ & $2(4 \%)$ \\
\hline Bacteroides & $5(10 \%)$ & 0 & \\
\hline Klebsiella & $3(6 \%)$ & $20(40 \%)$ & \\
\hline No growth & $6(12 \%)$ & \\
\hline
\end{tabular}

$P$ value derived by applying chi-square test

Table 4 shows the culture findings of the conjunctiva flora among the study subjects. The most common organism found in the culture among both the groups was Staphylococcus aureus but coagulase negative Staph. aureus was found to be more common among the diabetes group than the control group, similarly the growth of gram negative bacilli like E.coli, bacteroides and klebsiella were more common among the diabetes group than the control group and the difference was found to be statistically significant $(\mathrm{p}<.05)$.

Tabl-5: Antibiotic resistance pattern detected among the study subjects

\begin{tabular}{|l|c|c|c|}
\hline Antibiotic resistant pattern & Diabetes group $(\mathbf{n}=\mathbf{4 4})$ & Control group $(\mathbf{n = 3 0})$ & P value \\
\hline Only to erythromycin & $13(29.5 \%)$ & $14(46.6 \%)$ & $11(36.6 \%)$ \\
\cline { 1 - 3 } Erythromycin and Ampicillin & $21(47.7 \%)$ & $5(16.6 \%)$ & \multirow{2}{*}{$\mathbf{0 0 1}$} \\
\hline $\begin{array}{l}\text { Erythromycin, ampicillin and } \\
\text { Cephalosporins }\end{array}$ & $10(22.7 \%)$ & \\
\hline
\end{tabular}

$P$ value derived by applying chi-square test

Table 5 shows the antibiotic resistant pattern among the study subjects. The antibiotic for which the resistant pattern was assessed was erythromycin, ampicillin and cephalosporins. It is inferred from the table that resistant pattern for more than one antibiotic was found to be more common among the diabetes group than the control group and the difference was found be statistically significant $(\mathrm{p}<.05)$.

\section{Discussion}

The presence of bacteria on conjunctiva may result from the direct contact with the outside environment and the connection to the adjacent skin, and the different results of culture are greatly attributed to various factors such as environment, age, administration of antimicrobial agents, etc. [11] the use of antimicrobial agents contributes to the emergence of the new drug resistant strains [12].

Although the conjunctival flora forms a defensive barrier against infection, it also includes major pathogens of ocular infections. In healthy individuals, the conjunctival flora is frequently comprised of same microorganisms as the skin flora. Gram-positive bacteria constitute the main elements of bacterial floral, though the positive culture rate and microorganisms grown show diversity [13].

Higher rates of bacterial colonization are expected in situations that weaken the immune system such as diabetes, advanced age, and corticosteroid use [14].
However, it has been reported in the literature that infections that substantially suppress the immune system, in conjunctival cultures of diabetic patients. Suto et al. studied 579 individuals and found a positive culture rate of $39.2 \%$ with CONS as the major bacterial flora element [10].

In the same study, the rate of gram-negative bacteria was $5.9 \%$ and the most common gram-negative bacterium was Escherichia coli. In our study, grampositive bacteria were the major bacterial flora element and among the gram-positive cultures, Staphylococcus aureus was most common, in which Coagulase Negative Staphylococcus (CONS) was the second most common microorganism in the non-diabetic group and the first most common in the diabetic group. Similar to our study, Mehmet.

Adam et al. identified Staphylococcus aureus as the most common bacterial flora element in diabetic patients [7]. 


\section{Original Research Article}

We found a significant difference in bacterial isolation rate between the diabetic and non-diabetic groups. Furthermore, there was also a difference in positive culture growth frequency between the diabetes group and the control group ( $88 \%$ and $60 \%$, respectively).

Despite reports in the literature of differences in bacterial growth frequency between individuals with and without diabetes, few researchers had also found no such difference. Karimsab and Razak found a higher positive culture rate in their diabetic group compared to their non-diabetic group $(34 \%$ versus $24 \%$, respectively) [15].

Higher frequency of positive cultures has also been observed in PDR patients. Arbab et al. observed a positive culture rate of $75 \%$ in their PDR group compared to $20 \%$ in patients without retinopathy, and Staphylococcus epidermidis was the most common isolate [16]. In contrast, Suto et al. found no difference in the frequency of positive cultures between diabetic and non-diabetic patients and no relationship between positive culture frequency and the presence of diabetic retinopathy [10]. These conflicting results may be attributable to the differences in the DR rate between studies. The DR rates in the afore mentioned studies were $86.77 \%$ for Karimsab and Razak, [15] 74.8\% for Arbab et al. [16] and $8.29 \%$ for Suto et al [10] in our study, the rate of DR was $8 \%$.

Another factor that may affect the conjunctival flora is type of hypoglycemic therapy. In our study, most of patients were using only oral hypoglycemic agents and only $10 \%$ of the subjects were using insulin and in that all the patients had shown culture positive. Arbab et al. observed no relationship between hypoglycemic therapy and bacterial growth frequency and also found that the duration of diabetes had no effect on positive culture rates [16] Similarly, Martins et al. grouped patients by diabetes duration (more or less than 5 years) and found that the duration of diabetes had no effect on the frequency of positive cultures or the variety of flora bacteria [17]. Martins et al. also found that hypoglycemic therapy, age and gender had no effect on culture results [17]. In this study we aimed to compare the conjunctival flora of diabetic patients and healthy individuals. The most important result of our study is the higher frequency of gram-positive bacterial isolates, mostly coagulase negative Staphylococcus aureus (CONS) in the diabetic group. Rubio et al. evaluated the conjunctival flora of patients prior to cataract surgery and found that diabetic patients had a higher prevalence of Klebsiella pneumoniae and gram-negative diplococci than non-diabetic patients [18].
Philips and Tasman found that gram-negative bacteria account for the higher prevalence of endophthalmitis in diabetics compared to non-diabetics and that gramnegative microorganisms result in a poorer endophthalmitis prognosis. In a study of endogenic endophthalmitis by Lim et al. including 53 patients, gram-negative bacteria were detected in $54.38 \%$ of cases [19] Klebsiella pneumoniae was the most common gram-negative bacterial isolate $(45.61 \%)$ and diabetes was determined to be the most significant underlying risk factor. Similarly, gram-negative agents are noteworthy in other infections in diabetics.

Zhang et al. showed that the prevalence of gramnegative bacteria was four times higher in diabetics with chronic rhinosinusitis than in a control group [20]. In another study of patients with diabetic foot ulcers, gram-negative bacteria were isolated from $65.1 \%$ of positive cultures [21].

Gram-positive and gram-negative bacteria differ in their sensitivity to antibiotics. Coşkun et al. found that among conjunctival isolates of Staphylococcus aureus, 91.1\% were sensitive to ofloxacin and $86.6 \%$ to ciprofloxacin, while only $8.8 \%$ were sensitive to penicillin G; $28.8 \%$ of the isolates were methicillin-resistant Staphylococcus aureus and among these cultures, 38.5\% showed sensitivity to ofloxacin or ciprofloxacin [22]. In the same study, sensitivity of isolated Staphylococcus epidermidis cultures to ofloxacin and ciprofloxacin was $92.5 \%$ and $91.5 \%$, respectively.

In our study we found majority (77\%) of the study subjects in the diabetes group showed resistant to ampicillin and erythromycin and a few (23\%) of them showed resistance to cephalosporins than that of the control group. Suto et al. found a higher prevalence of methicillin-resistant CONS in diabetic patients and reported resistance rates of $14 \%$ to levofloxacin and $17.9 \%$ to tobramycin in the isolates they obtained [10]. Gupta et al. found that in all gram-positive cultures isolated from endophthalmitis cases were sensitive to vancomycin and all gram-negative cultures were sensitive to ceftazidime [23].

Long et al. investigated endophthalmitis following trauma and found the prevalence of gram-negative to be 29.1\%, with Pseudomonas aeruginosa and Escherichia coli as the most common isolates [24]. Furthermore, due to the increasing frequency of multiple antibiotic resistance in gram-negative bacteria, they recommended using ciprofloxacin, tobramycin and cephalosporin together in cases of Pseudomonas aeruginosa-related endophthalmitis. 


\section{Original Research Article}

It was thought that normal ocular flora could be nonpathogenic or occasionally pathogenic. However, the pathogens of some bacterial endophthalmitis, bacterial corneal ulcers, blepharitis, conjunctivitis, and other ocular infection diseases turned out to consistent with conjunctival isolated bacteria, and the $S$. epidermidis has become the predisposing pathogen [25].

Although, the preoperational topical antibiotics can hardly make the conjunctival sacs sterile, they can effectively reduce the isolating bacteria [26].

The susceptibility of each antibiotic to different bacteria is not identical, and the regional reported drug resistance varies widely due to different environment and the use of antibiotics [27].

Evolving bacterial resistance represents one of the most serious global public health problems, and overcoming this problem has become a great challenge. Due to different ethnic group, environment and antimicrobial therapy, the distribution and resistant profiles of conjunctival bacteria vary significantly from area to area.

Therefore, the investigation in these subjects can be clinically useful in the primary empirical antimicrobial strategy before knowing the laboratory results.

\section{Conclusions}

Bacterial growth in the conjunctiva was found to be common in both diabetes and non-diabetes people. Organisms such as coagulase negative staphylococcus (CONS), gram negative bacteria such as E.coli, Klebsiella and Bacteroides were found to be more common among the diabetes patients than that of nondiabetes people.

Though few studies had reported that there are no significant changes in the conjunctiva flora among the diabetes and non-diabetes group, our study has shown a difference in the conjunctiva flora between these two groups.

One of the limitations of the present study is the small sample size, so similar type of studies with a larger sample has to be conducted to prove that there is a difference in the conjunctiva flora between diabetes and non-diabetes group.

Contribution of this study: Considering that flora elements may be important pathogens in ocular infections, treatment approaches to gram-negative bacteria should not be ignored in cases of ocular infections in diabetics.

Acknowledgments: The author would like to thank the head of the department, professor, associate professor, assistant professor, postgraduates and optometry department staff of Vinayaka Mission's Kirupanada Variyar Medical College and Hospital, Salem, Tamil $\mathrm{Nadu}$, for helping with data collection and laboratory analyses to complete the research work.

Funding: Nil, Conflict of interest: Nil Permission from IRB: Yes

\section{Ethical Committee Approval Given By Institution}

\section{References}

1. Eye Health. Conjunctiva. American Academy of Ophthalmology, March-28, 2018.

2. Bethesda. Tears. Medline Plus, October-23, 2018.

3. Labtests online. Normal Flora. American Association for Clinical Chemistry, July-10, 2017.

4. UK ESSAYS. Normal Flora and their Benefit Feb$22,2017$.

5. Ravee Ratnumnoi, Narumon Keorochana, and Chavalit Sontisombat. Normal flora of conjunctiva and lid margin, as well as its antibiotic sensitivity, in patients undergoing cataract surgery at Phramongkutklao Hospital", Clin Ophthalmol. 2017; 11: 237-241, doi:10.2147/OPTH.S109247

6. Saiba Eugene Semanyenzi. Normal Conjunctival Flora as seen in Adult Patients at Kigali University Teaching Hospital. Rwanda Medical Journal, June70(2): 22-24.

7. Adam M, Balcı M, Bayhan HA, et al. Conjunctival Flora in Diabetic and Nondiabetic Individuals. Turk J Ophthalmol. 2015 Oct;45(5):193-196. Epub 2015 Oct 5.

8. New biosensor could monitor glucose levels in tears and sweat. American Chemical Society (ACS) News Service Weekly Press Pac: Wed Jan 24 12:30:47 EST 2018.

9. Kumar MA, Kurien SS, Selvaraj S, Devi SU, Selvasundari S. Comparison of different techniques of cataract surgery in bacterial contamination of the anterior chamber in diabetic and non-diabetic population. Indian J Ophthalmol 2012;60:41-4. 


\section{Original Research Article}

10. Suto C, Morinaga M, Yagi T, et al. Conjunctival sac bacterial flora isolated prior to cataract surgery. Infect Drug Resist. 2012;5:37-41. doi: 10.2147/IDR. S27937. Epub 2012 Jan 24.

11. Sharma PD, Sharma N, Gupta RK, et al. Aerobic bacterial flora of the normal conjunctiva at high altitude area of Shimla Hills in India: a hospital based study. Int J Ophthalmol. 2013 Oct 18;6(5):723-6. doi: 10.3980/ j.issn. 2222-3959.2013.05.32. eCollection 2013.

12. WHO. Antimicrobial resistance. 15 February, 2018.

13. Kenneth Todar. The Normal Bacterial Flora of Humans. Todar's Online Textbook of Bacteriology. The Good, the Bad, and the Deadly Page-3, 2012

14. Afsun Sahin et al. Changes in the conjunctival bacterial flora of patients hospitalized in an intensive care unit. Arq. Bras. Oftalmol. vol.80 no.1 São Paulo Jan./Feb. 2017

15. Karimsab D, Razak SK. Study of aerobic bacterial conjunctival flora in patients with diabetes mellitus. Nepal J Ophthalmol. 2013 Jan-Jun;5(1):28-32. doi: http://dx.doi.org/10.3126/nepjoph.v5i1.7818.

16. Arbab TM, Qadee S, Iqbal S, Mirza MA. Aerobic bacterial conjunctival flora in diabetic Patients. Pak J Ophthalmol. 2010;26:177-181.

17. Martins EN, Alvarenga LS, Höfling-Lima AL, et al. Aerobic bacterial conjunctival flora in diabetic patients. Cornea. 2004 Mar;23(2):136-42.

18. Fernández-Rubio ME, Rebolledo-Lara L, MartinezGarcía M, et al. The conjunctival bacterial pattern of diabetics undergoing cataract surgery. Eye (Lond). 2010 May;24(5):825-34. doi: 10.1038/eye.2009.218. Epub 2009 Aug 28.

19. Lim HW, Shin JW, Cho HY, et al. Endogenous endophthalmitis in the Korean population: a six-year retrospective study. Retina. 2014 Mar;34(3):592-602. doi: 10.1097/IAE.0b013e3182a2e705.
20. Zhang Z, Adappa ND, Lautenbach E, et al. The effect of diabetes mellitus on chronic rhinosinusitis and sinus surgery outcome. Int Forum Allergy Rhinol. 2014 Apr;4(4):315-20. doi: 10.1002/alr.21269. Epub 2014 Jan 10 .

21. Shanmugam P, M J, Susan S L. The bacteriology of diabetic foot ulcers, with a special reference to multidrug resistant strains. J Clin Diagn Res. 2013 Mar;7(3):441-5. doi: 10.7860/JCDR/2013/5091.2794. Epub 2013 Mar 1.

22. Coşkun M, Koçak AG, Simavlı H, Anayol MA, Toklu Y, Çelikbilek N. Analyzing normal conjunctival flora and detecting antibiogram sensitivity to flouroquinolones and penicillin derivates. Glo-Kat. 2007;2:167-170.

23. Gupta A, Orlans HO, Hornby SJ, et al. Microbiology and visual outcomes of culture-positive bacterial endophthalmitis in Oxford, UK. Graefes Arch Clin Exp Ophthalmol. 2014 Nov; 252(11):1825-30. doi: 10.1007/s00417-014-2658-7. Epub 2014 Jun 11.

24. Long $\mathrm{C}$, Liu B, Xu C, Jing $\mathrm{Y}$, et al. Causative organisms of post-traumatic endophthalmitis: a 20-year retrospective study. BMC Ophthalmol. 2014 Mar 25;14:34. doi: 10.1186/1471-2415-14-34.

25. Carreras B. Bacteriological analysis in the management of conjunctivitis. Comparison of antibiotic resistance between 1982 and 2008. Arch. Soc. Esp. Oftalmol. 2012;87, 107-111

26. Mshangila B, Paddy $M$, Kajumbula $H$, et al. External ocular surface bacterial isolates and their antimicrobial susceptibility patterns among preoperative cataract patients at Mulago National Hospital in Kampala, Uganda. BMC Ophthalmol. 2013 Nov 15; 13:71. doi: 10.1186/1471-2415-13-71.

27. Muluye D, Wondimeneh Y, Moges F, et al. Types and drug susceptibility patterns of bacterial isolates from eye discharge samples at Gondar University Hospital, Northwest Ethiopia. BMC Res Notes. 2014 May 12;7:292. doi: 10.1186/1756-0500-7-292.

\section{How to cite this article?}

Rajeshkannan R., Venkatesan M.J., Ezhilvendhan K., Rao A.V.R. A study to compare the conjunctival flora of nondiabetic individuals with that of diabetic patients. Trop J Ophthalmol Otolaryngol.2019;4(1):4854.doi:10.17511/jooo.2019.i01.10 